\title{
Proteomic analysis of laser capture microscopy purified myotendinous junction regions from muscle sections
}

Tugba Can ${ }^{1}$, Laura Faas ${ }^{1}$, David A Ashford ${ }^{2}$, Adam Dowle², Jerry Thomas ${ }^{2}$, Peter O'Toole ${ }^{2}$ and Gonzalo Blanco ${ }^{* *}$

\begin{abstract}
The myotendinous junction is a specialized structure of the muscle fibre enriched in mechanosensing complexes, including costameric proteins and core elements of the z-disc. Here, laser capture microdissection was applied to purify membrane regions from the myotendinous junctions of mouse skeletal muscles, which were then processed for proteomic analysis. Sarcolemma sections from the longitudinal axis of the muscle fibre were used as control for the specificity of the junctional preparation. Gene ontology term analysis of the combined lists indicated a statistically significant enrichment in membrane-associated proteins. The myotendinous junction preparation contained previously uncharacterized proteins, a number of z-disc costameric ligands (e.g., actinins, capZ, aB cristallin, filamin C, cypher, calsarcin, desmin, FHL1, telethonin, nebulin, titin and an enigma-like protein) and other proposed players of sarcomeric stretch sensing and signalling, such as myotilin and the three myomesin homologs. A subset were confirmed by immunofluorescence analysis as enriched at the myotendinous junction, suggesting that laser capture microdissection from muscle sections is a valid approach to identify novel myotendinous junction players potentially involved in mechanotransduction pathways.
\end{abstract}

\section{Background}

Laser-assisted cell microdissection in combination with laser-pressure catapulting (commonly referred to as laser capture microdissection or LCM) has been exploited for over a decade to isolate pure population of cells, specific regions from tissue sections or even single chromosomes [1-4]. LCM has also been applied to identify the unique expression profiles of specialized regions within complex cells. For example, LCM has been combined with RNA isolation and transcriptome analysis to identify specific transcripts and components of the neuromuscular junction $[5,6]$. In combination with liquid chromatography tandem mass spectrometry (LC-MS/MS), LCM has been extensively used, amongst others applications, to purify and profile cancer cells (for a review see [7]), profile plaques in neurological disease [8-11] or elucidate the expression profile of inclusion bodies within muscle fibres [12]. In the experimental process of an expression profiling, LCM is often

\footnotetext{
* Correspondence: gonzalo.blanco@york.ac.uk

'Department of Biology, University of York, Wentworth Way, York YO10 5DD, UK

Full list of author information is available at the end of the article
}

the limiting step, given the length of time required to recover a small amount of material. However, when combined with high-resolution mass spectrometers, LCM of tissue sections can potentially identify hundreds of proteins from as little as 1000 cells [13].

In an attempt to identify candidate proteins for mechanotransduction processes, we decided to focus on the myotendinous junction (MTJ). Like the neuromuscular junction, the myotendinous junction is also a highly specialized anatomical region of the muscle fibre thought to be controlled by specialized underlying nuclei. The MTJ encompasses an alignment of protein complexes from the subsarcolemmal cytoskeleton, through the sarcolemma and basal lamina, to the collagen matrix on the tendinous side of the junction (see [14] for a recent review on the development and organization of the MTJ). Actin filaments bundled with alphaactinin and desmin project from the electrodense terminal sarcomeric z-discs [15] towards the sarcolemma. At the sarcolemma, they interact with the dystrophin-associated and the $\alpha 7 \beta 1$ integrin protein complexes, which in turn connect with the extracellular matrix through the basal lamina protein laminin, following a similar arrangement to 
the costamere [16-20]. The MTJ is thus a major site of force transmission from myofibrils to the extracellular matrix and adapts to mechanical stress by increasing the muscle-tendon contact area [21]. Indeed, the myotendinous junction is enriched in costameric proteins and core elements of the z-disc.

In recent years, the sarcomeric z-disc has emerged as a plausible structure that mediates adaptive responses to mechanical stresses. Such notion has evolved from the discovery of proteins over the last two decades that, when mutated or lost from the z-disc, provoke skeletal muscle and heart disorders. Characterizations of the muscle phenotypes in these disorders have demonstrated that the $\mathrm{z}$-disc is not just a structural link between sarcomeres, but that it contains both a structural scaffold for modulating biological sensors [22] and signalling hubs for mechanosensation and mechanotransduction [23,24]. Moreover, the presence within the z-disc of the kyphoscoliosis peptidase protein KY, a protein required for the muscle hypertrophic response to chronic overload [25,26], suggests that the zdisc could also contain mechanistic triggers of hypertrophy.

Although the z-disc would be an ideal structure to profile in order to identify new players of mechanoreception and transduction, purification of z-discs that retain their intact physiological interactions is a challenging task. Biochemical enrichments of the $\mathrm{z}$-disc have been successfully done using strong treatments (e.g., [27]). While these preparations have been useful to elucidate the cytoarchitecture of the purified insoluble material, most of the soluble associated proteins are lost during the extraction process, therefore defeating the purpose of a follow up proteomic analysis. On the other hand, purification of the intact $\mathrm{z}$-disc by laser capture microdissection using thick longitudinal sections of muscle fibres is not possible because the width of the z-band is only $30-100 \mathrm{~nm}$ in the vertebrate muscle fibre [28], which is far beyond the resolution of LCM technology. As a potential alternative, we evaluate here the ability of LCM combined with LC-MS/MS analysis to profile the myotendinous junction region.

\section{Results}

\section{LCM based purification of membrane sections}

Mouse muscles that were processed included gastrocnemius, soleus, extensor digitorium longus, tibialis anterior and biceps. Fresh frozen tissue sections were used, instead of formalin-fixed and paraffin-embedded samples, to avoid any confounding factor that might be introduced by unequal fixation of the tissues. Mounting of the frozen muscles and processing by laser capture microdissection was carried as described in Methods. To facilitate the identification of the transition from the myofibre to the collagen matrix, a mild hematoxylin and eosin staining (H\&E) was applied on sections spanning the myotendinous junctions (Figure 1A). This step also rendered the sections slightly drier and facilitated UV absorption, in our hands resulting in much more efficient catapulting of the tissue cuts from the slide into the collection tube. Given the narrow sarcolemmal regions selected for LCM purification (see a representative cut in Figure 1A, panels C and D), collecting enough protein for successful proteomic analysis would have been impractical using the recommended section thickness $(8-12 \mu \mathrm{m})$. Therefore, sections ranging from $12 \mu \mathrm{m}$ to $30 \mu \mathrm{m}$ were tested for their ability to be catapulted into the collection tubes following laser dissection. Under the fixation and staining protocol applied, sections of up to $20 \mu \mathrm{m}$ could be efficiently catapulted. Energy levels and focus of the laser were also tested and adjusted to the specific regions (see Methods for details). In total, 800 cuts were collected from the myotendinous junction and a similar amount from the extra junctional membrane regions, hereafter referred to as samples MTJ and $M$, respectively.

\section{Proteomic processing}

The sectioned areas were as restricted as possible to the membrane (Figure 1A), therefore yielding very limited material that could not be subjected to further biochemical fractionations. Instead, all of the collected materials from samples MTJ and M were resuspended in SDS sample buffer and run into a polyacrylamide gel, which was subsequently Coomassie stained and cut into sections as illustrated in Figure 1B. Protein identification was performed by LC-MS/MS (see Methods for details). A total of 405 proteins (excluding keratins) were identified which included unique and common hits to samples MTJ and M. These identifications were classified as 44 IDs unique to the myotendinous junction, 159 IDs unique to the peripheral membrane and 202 IDs common to both preparations (Figure 1C). Table 1 shows a selection of proteins identified in the MTJ sample. The full lists are presented in Additional file 1: Table S1.

As expected, the list of proteins common to MTJ and $M$ contained extracellular matrix constituents (collagens I and VI), proteins involved in extracellular matrix assembly (biglycan, fibromodulin, mimecan), anchoring of the basement membrane (prolargin, laminin), transmembrane anion channels (Vdac 1, 2 and 3) and cytoskeletal anchoring proteins (e.g., vimentin). Achievement of specificity was suggested by the fact that most hits in the full lists were proteins with a function in muscle. To further test whether LCM purification resulted in the intended enrichment of intracellular and extracellular membraneassociated proteins, we searched for evidence of statistically significant enrichment of GO terms in the MTJ list. To avoid biasing the analysis towards membrane associated extracellular components, all keratins were eliminated from the list, as they were presumed to be contaminants. The 246 hits were mapped to 215 Mus musculus supervised 
A
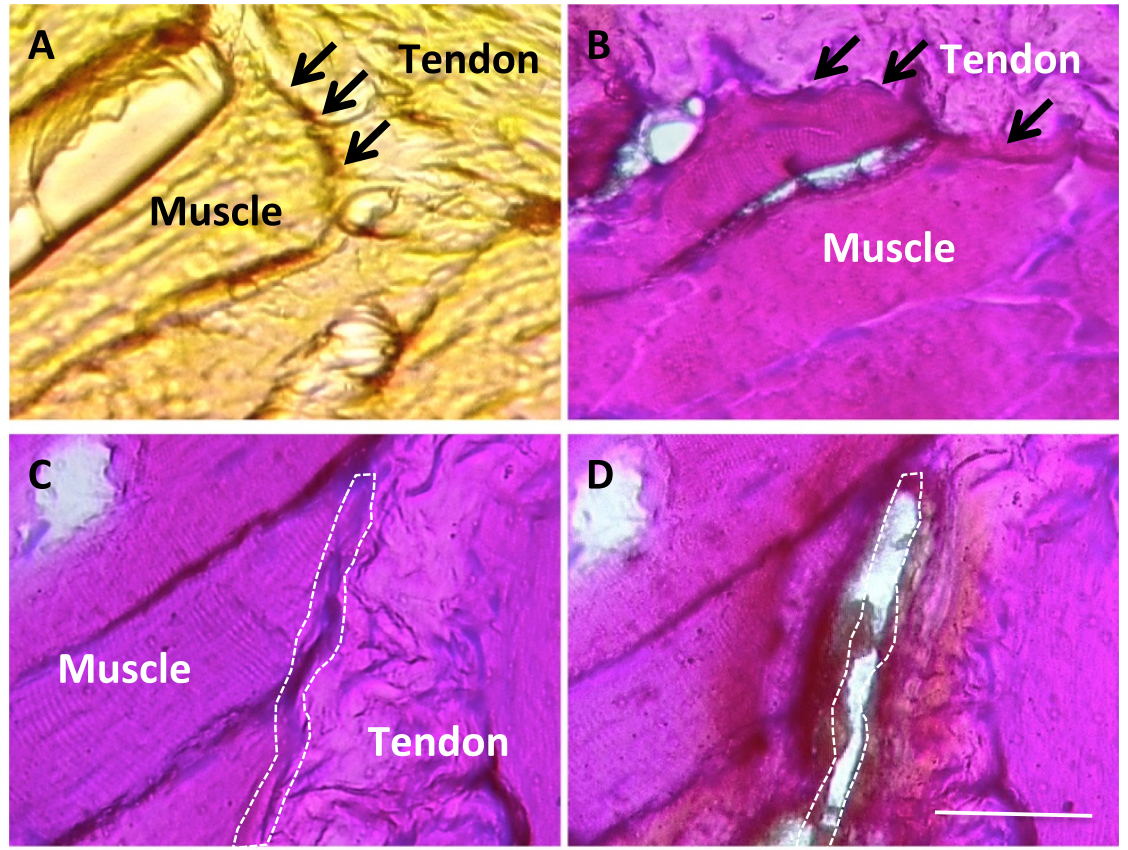

B

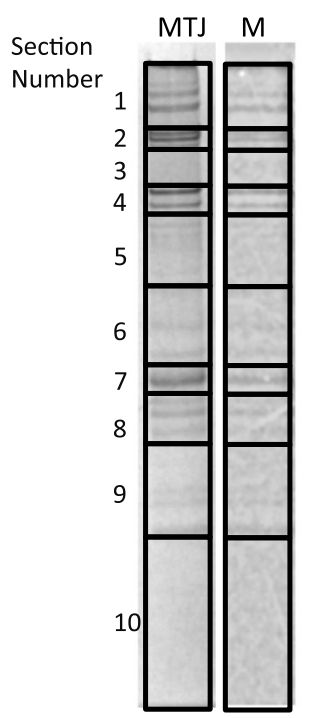

C

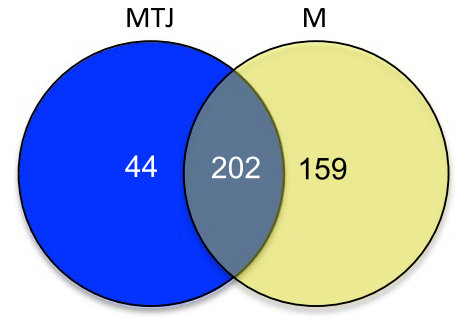

Figure 1 Proteomic analysis of laser capture microdissection of myotendinous junction regions. A) A comparative view of the myotendinous junction of a non-stained section (panel A) and an H\&E stained section (panel B), with tendon and muscle regions identified as indicated and myotendinous regions identified by arrows. Panels $C$ and $D$ show a representative example of a myotendinous junction selected region (white dotted line) selected for microdissection before (panel C) and after (panel D) laser application and catapulting. All pictures taken with a 40X objective. Scale bar indicates 50 microns. B) Protein gel illustrating sections excised for LC-MS/MS analysis. The grid overlaying the lanes of solubilized material from myotendinous region (MTJ) or extrajunctional membrane (M) indicates the gel blocks used for in-gel tryptic digestions. C) A Venn diagram indicating the number of proteins identified according to their presence in the myotendinous junction (MTJ) or the extrajunctional membrane region (M).

entries in UniProtKB (http://www.uniprot.org/). This list was then submitted to the software package GOrilla [29], which confirmed that 211 out of the 215 genes were associated with GO terms. Finally, computerized analysis of the GO terms, using as reference the reviewed Mus musculus uniprot protein list (16665 proteins), showed that several categories corresponding to extracellular, membrane bound, $\mathrm{z}$-disc and mitochondrial associated were indeed enriched at $p$ values ranging between $10^{-5}$ and $10^{-43}$ (Additional file 2: Figure S1; full lists of genes per enriched GO term with the corrected $p$ values for multiple testing [30] are shown in Additional file 3: Table S2). Analysis of the list 
Table 1 Selection of proteins identified in the MTJ sample

\begin{tabular}{|c|c|c|c|c|c|}
\hline \multicolumn{3}{|c|}{ Connective tissue } & \multicolumn{3}{|c|}{ Transmembrane } \\
\hline Protein & Accession & Mascot score & Protein & Accession & Mascot score \\
\hline Biglycan & IPI00123194 & 396 & Vdaxcl & IPI00230540 & 1135 \\
\hline Fibromodulin & IPI00120187 & 1487 & Vdac2 & IPI00122547 & 116 \\
\hline Mimecan & IPI00120848 & 96 & Vdac3 & IPI00122548 & 413 \\
\hline Col1a2 & IPI00222188 & 3023 & Mpz & IPI01008333 & 102 \\
\hline Col6a3 & IPI00830749 & 1012 & & & \\
\hline Col6a2 & IPI00621027 & 192 & & & \\
\hline Col1a1 & IPI00329872 & 1357 & & & \\
\hline Col6a1 & IPI00339885 & 861 & & & \\
\hline Col12a1 & IPI00121430 & 767 & & & \\
\hline Prolargin & IPI00122293 & 718 & & & \\
\hline Laminin & IPI00756745 & 33 & & & \\
\hline \multicolumn{3}{|c|}{ Intracellular membrane associated } & \multicolumn{3}{|c|}{ Z-disc/costameric ligands } \\
\hline Protein & Accession & Mascot score & Protein & Accession & Mascot score \\
\hline Vinculin & IPI00405227 & 18 & Actinin & IPI00136701 & 4955 \\
\hline Plectin & IPI00229509 & 249 & CapZ & IPI00111265 & 192 \\
\hline Desmin & IPI00130102 & 1462 & a-cristallin & IPI00138274 & 93 \\
\hline Plakoglobin & IPI00229475 & 273 & FilaminC & IPI00664670 & 138 \\
\hline Desmoplakin & IPI00553419 & 16 & Cipher & IPI00403040 & 547 \\
\hline \multirow[t]{9}{*}{ Asap2 } & IPI00355808 & 16 & FHL1 & IPI00309997 & 18 \\
\hline & & & Telethonin & IPI00119331 & 24 \\
\hline & & & Nebulin & IPI00720238 & 76 \\
\hline & & & Titin & IPI00986455 & 19546 \\
\hline & & & Enigma-like & IPI00415684 & 108 \\
\hline & & & Myotilin & IPI00120508 & 50 \\
\hline & & & Telethonin & IPI00119331 & 24 \\
\hline & & & Myoxenin-2 & IPI00122334 & 18 \\
\hline & & & a-actin & IPI00114593 & 19409 \\
\hline
\end{tabular}

specific to the $M$ sample produced identical categories of GO term enrichment (data not shown).

\section{Identification of myotendinous junction proteins}

To assess the quality of the myotendinous membrane preparation we looked for the presence of costameric proteins [20]. As summarized in Table 1, many costameric proteins were indeed present, including members of the connective tissue (collagens, laminin, prolargin, fibromodulin), intracellular (vinculin, g-actin, plectin, desmin) and a number of z-disc costameric ligands (actinins, CapZ, $\alpha B$ crystallin, filamin $C$, cypher, calsarcin, FHL1, telethonin, nebulin, titin and an enigma-like protein). Thus, the preparation from the junctional region contained a good representation of proteins integral to the costameric network. In addition, the myotendinous junction preparation contained unique hits to: components of desmosomes (plakoglobin), extracellular proteins (Alpha-2-HS-glycoprotein, Lyz1 Lysozyme C-1), proteins associated to the cytosolic face of the plasma membrane (the two annexin proteins Anxa1 and Anxa6) and an integral membrane protein (myelin). Many proteins were identified only in the M preparation (e.g., $\alpha$-syntrophin, $\alpha$-sarcoglycan) but, intriguingly, other transmembrane proteins of the dystrophin associated protein complex were absent from both preparations, perhaps reflecting a limitation of the non-fractionated approach undertaken (see also Discussion).

\section{Expression patterns of MTJ candidates}

Many of the proteins identified have been shown to have expression at sarcomeric or costameric level, but no information was available regarding their expression at the 

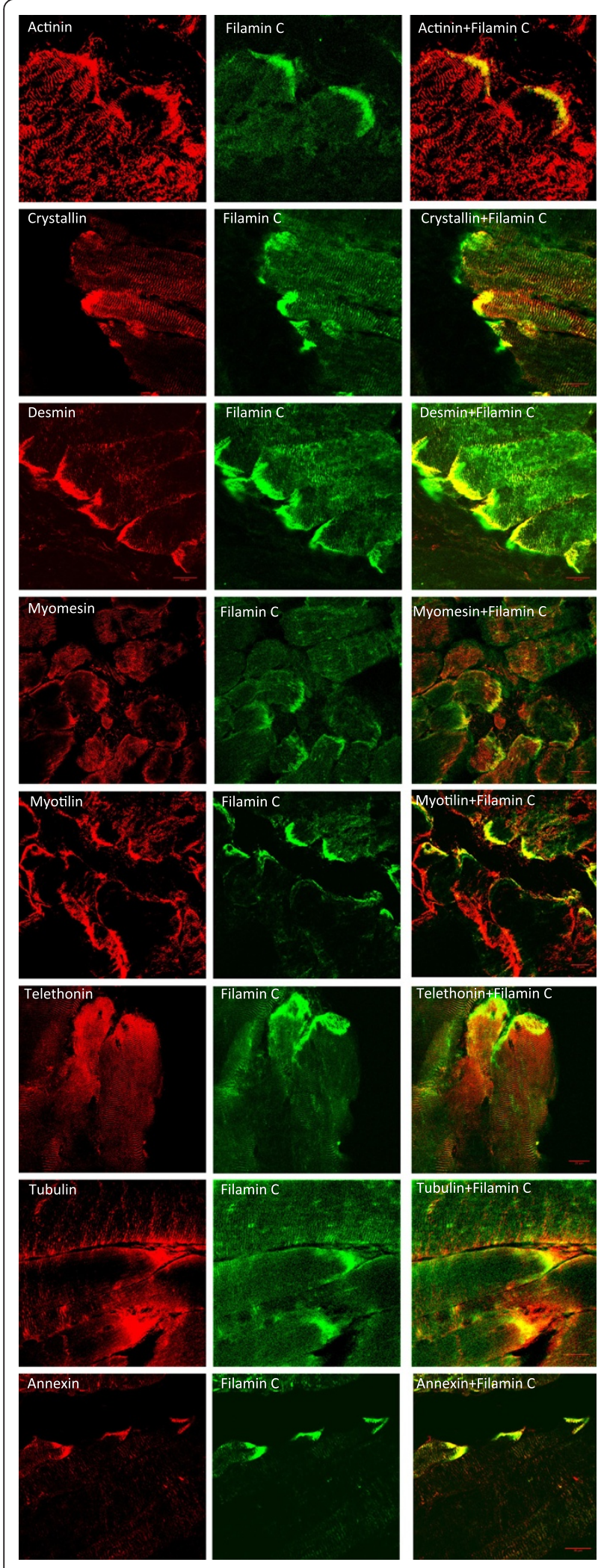

Figure 2 Immunostainings of selected proteins from the MTJ sample. Longitudinal sections of tibialis anterior muscle from 45 days old C57/Bl6 male were mice stained with antibodies against the indicated proteins. Strong staining of filamin $C$ identifies the myotendinous junction region in each case. All pictures taken with a $63 x$ oil lense. The scale bar indicates 20 microns.

myotendinous junction. To confirm the quality of the results above, we tested the expression of some of the proteins identified by immunofluorescence for which commercial or published antibodies exist. Localization at the myotendinous junction was tested by co-localization with the well-established marker filamin $C$ [31]. The results shown in Figure 2 indicates that for $\alpha$-actinin, $\alpha B$ crystallin, desmin, myomesin, myotilin, telethonin, tubulin and annexin I (see Methods for antibody details), the expression was consistent with the proteomic results, since a good degree of co-localization with filamin C was obtained for all of them. Localization at the MTJ was anticipated for desmin [32] and $\alpha$-actinin [33]. However, a stronger signal for $\alpha$-crystallin, myomesin, myotilin, telethonin and annexin $I$ at the myotendinous junction region had not been, to our knowledge, previously reported. Additionally, we tested three proteins that were identified in the M sample only: the giant sarcomeric protein titin 1 and the dystrophin associated protein complex members $\alpha$-sarcoglycan (transmembrane) and $\alpha$-syntrophin (cytoplasmic) (Figure 3). Titin 1 appeared to show very little co-localization with filamin C. In contrast, $\alpha$-syntrophin and $\alpha$-sarcoglycan gave stronger signal at the myotendinous junction compared to the extra junctional membrane. This was expected given that syntrophins and sarcoglycans are members of the dystrophin associated protein complex and dystrophin has been previously found at subsarcolemmal deposits at the junctional folds of the myotendon [34,35].

Myomesin 1, 2 and 3 are major components of the $\mathrm{M}$-band [36,37], therefore the presence of the three of them in the MTJ sample was unexpected. To distinguish between accumulations at the MTJ junctional folds from increased expression of the candidate protein at the terminal sarcomeres, higher resolution confocal views were obtained. The results shown in Figure 4 indicate that desmin, $\alpha \mathrm{B}$ crystallin, annexin I and $\alpha$-syntrophin appear to modify their expression pattern at this level and strongly stain the MTJ region. In contrast, as determined by costainings with $\alpha$-actinin (data not shown), telethonin and myomesin remained restricted to the $\mathrm{z}$-disc and the M-line, respectively (Figure 4). Thus, the presence of telethonin and myomesin in the MTJ sample may originate from their increased expression at the most terminal sarcomeres. Incubations of the sections with the secondary antibodies alone did not produce any detectably signal using identical incubation and confocal setting conditions. 

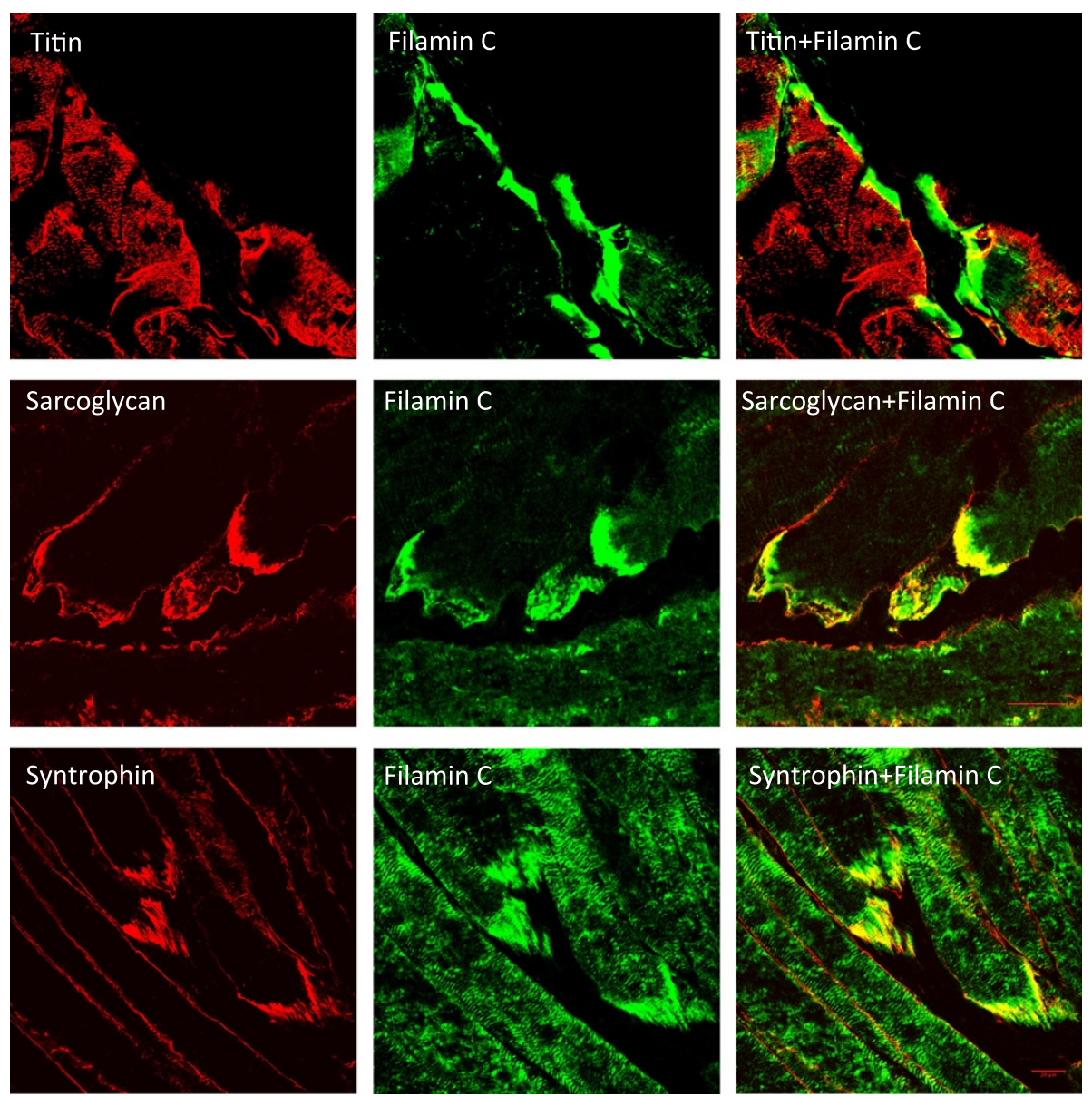

Figure 3 Co-localization analysis of three proteins that were identified in the membrane sample only. Titin shows very little co-localization with the myotendinous junction marker filamin C. The membrane-associated proteins a-sarcoglycan and a-syntrophin show partial co-localization. All pictures taken with a $63 x$ oil lense. The scale bar indicates 20 microns.

\section{Discussion}

The skeletal muscle mechanoreceptors and how specific mechanical inputs are converted into biochemical signals that trigger specific muscle adaptations remain elusive. In recent years, the sarcomeric $\mathrm{z}$-disc has emerged as a plausible structure that mediates adaptive responses to mechanical stresses (e.g.,: $[38,39])$. This study aimed at testing the suitability of LCM from muscle sections in combination with LC/MS-MS for the identification of proteins associated with the myotendinous junction, since this structure contains z-disc material and is physically more approachable than the $\mathrm{z}$-disc. Although there are obvious limitations to this approach, e.g., restriction of the analysis to soluble proteins or the physical resolution of the cuts, the proteomic analysis of the myotendinous junction and extra junctional membrane content showed a significant enrichment of GO-terms for intracellular and extracellular membrane-associated proteins. Indeed, despite $H \& E$ staining having been suggested to interfere with direct MALDI MS analysis of LCM captured cells [40], the protocol as described here was successful in identifying some differences between both membrane regions. Moreover, many costameric/z-disc proteins were identified in the myotendinous junction sample. The most likely reason why specific proteins previously found associated with the myotendinous junction were not identified here is that they were not present in sufficient abundance in the solubilized sample. For example, collagen VI, a protein enriched at the muscle endomysium [41], was successfully identified, but the less abundant collagen $\mathrm{V}$ [42] failed to be detected. Limited solubility or the fact that trypsin digestion may have also resulted in peptides outside the optimal size range or that do not ionise well and therefore are not suitable for MS, might explain why additional members of the costamere junction or other known MTJ proteins failed to be detected. It is also anticipated, given the size of the myotendinous junctional folds relative to the purified LCM cuts, that many of the identified proteins will not show a specific subcellular myotendinous junction localization. 

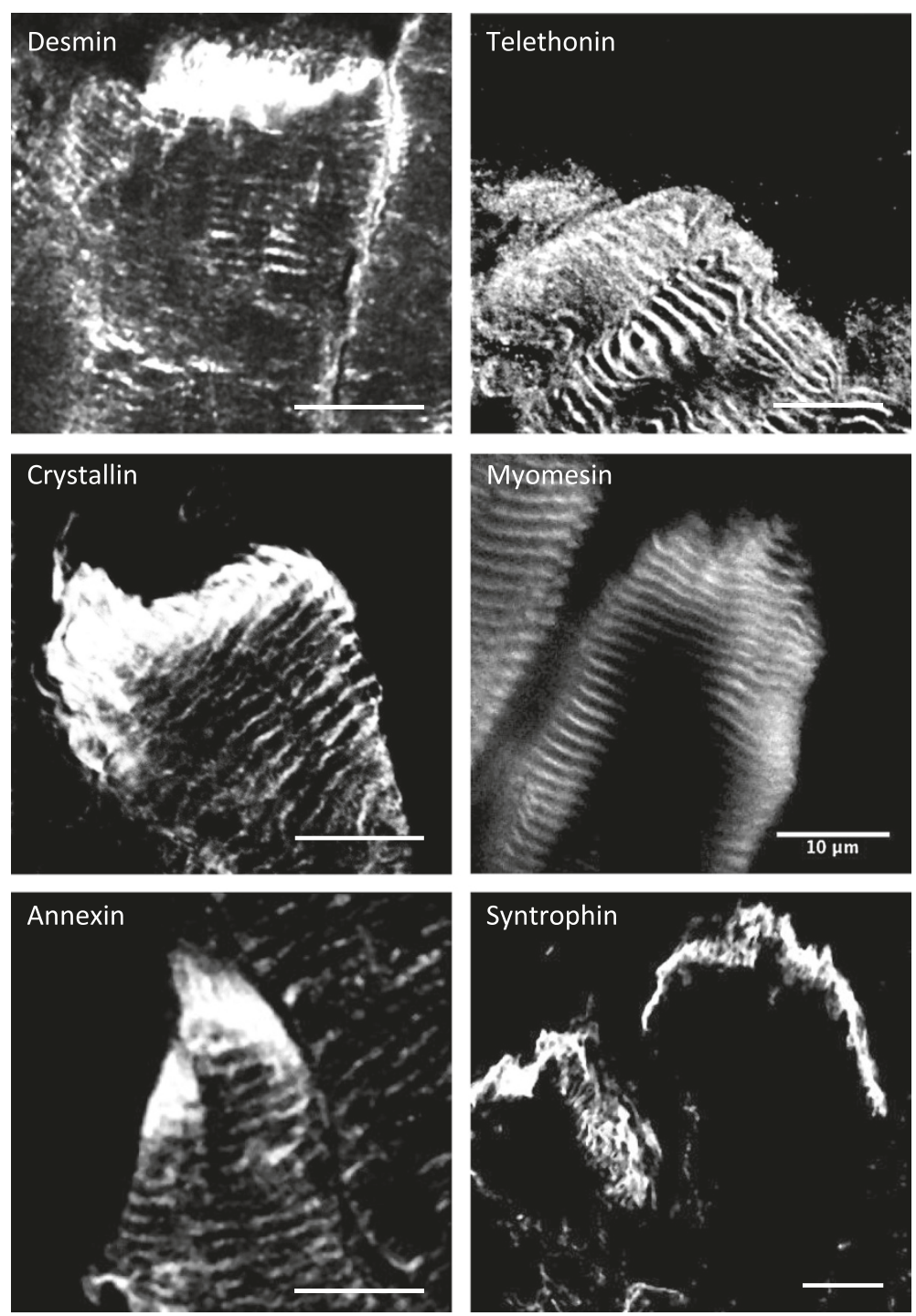

Figure 4 Changes in subcellular localization patterns from the myofibre to the MTJ region illustrated for selected proteins. Note that desmin, aB crystallin and anexin I appear to fill the NMJ region on the muscle side beyond their z-disc localization. Telethonin (z-disc) and myomesin ( $\mathrm{M}$ line) appear to increase their concentration at the most terminal sarcomeres without changing their characteristic banding patterns. For comparison, the MTJ pattern of a-syntrophin is also shown. All pictures taken with a $63 x$ oil lense with variables zoom factors applied. All scale bars indicate 10 microns.

A mechanotransduction mechanism for maintaining homeostasis in mechanically stressed cells has recently been proposed involving tension-induced targeted degradation of the actin crosslinker protein filamin and its upregulation $[43,44]$. Filamin $C$ is highly enriched at the myotendinous junction and its ability to crosslink makes it a suitable candidate to sense mechanical stress, as tension can induce the exposition of cryptic interaction sites [45] and changes in the rates of protein turnover [43]. Since the immunoglobulin- and fibronectin-like repeats present in filamin are also found in many other muscle proteins with filament and myofibril crosslinking roles $[46,47]$, adaptations to specific mechanical strains could also be mediated by other globular repeat containing proteins. In addition to filamin $\mathrm{C}$, other proteins identified in the MTJ sample containing a succession of repeated domains included titin, nebulin, myosin binding protein $\mathrm{C}$, obscurin, myotilin and the three myomesin isoforms. The presence of the three known M-band myomesin isoforms was intriguing. Myomesins share repeats of globular domains in their composition, have been proposed to participate in a stress-sensing mechanism and provide elasticity to the M-band $[36,48,49]$. Although myomesin accumulation at the myotendinous junction appears to be the case on the basis of the strong immunofluorescence signal detected at the edge of the muscle fibre, the signal remained 
restricted to the M-band at the terminal ends of the myofibrils (Figure 4). It would therefore be unlikely for myomesin to participate in membrane bound stretch complexes at the myotendinous junction, but it is plausible that their increased expression at this region reflects higher mechanical stress of the terminal sarcomeres. The same might be concluded for telethonin, as the stronger signal observed at the end of the fibre was not caused by accumulation beyond its characteristic z-disc expression. We therefore conclude that telethonin and myomesin do not have myotendinous junction specific functions, but that their presence in the proteomic MTJ sample is probably due to their increased expression at the most terminal sarcomeres.

Three proteins of diverse functions that have been previously associated with the $\mathrm{z}$-disc were shown here to intensely stain the MTJ: myotilin, annexin I and $\alpha B$ crystallin. Myotilin is a z-disc protein that binds F-actin directly and bundles actin filaments in vitro [50]. However, despite the fact that mutations in the myotilin gene have been implicated in limb girdle muscular dystrophy 1A (LGMD1A) [51], myofibrillar myopathy (MFM) [52], and in a rare condition called spheroid body myopathy (SBM) [53], the function of myotilin in normal muscle physiology remains unclear. Annexins are structurally related calcium dependent phospholipid binding proteins with ability to promote contact between vesicle membranes [54]. The proteomic lists contained annexin VI (common to the MTJ and $M$ samples) and annexin I (unique to the MTJ sample). Annexin VI has been involved in the maintenance of the cytoskeleton and extracellular matrix integrity [55] and appears to co-localize with $\alpha$-actinin at z-discs in cardiomyocytes [56]. Annexin I is expressed in satellite cells of adult muscle [57], but a function in muscle fibre has not been reported. Immunofluorescence with Annexin I antibodies showed very high expression at the MTJ of adult muscle. Given that annexin I has been shown to interact with profilin [55], a crucial protein in the actin polymerization process, it is plausible that annexin I contributes to cytoskeletal remodeling at this localization. $\alpha \mathrm{B}$ crystallin is a member of the small heat shock protein family [58] and acts as a molecular chaperone. In skeletal muscle, $\alpha B$ crystallin localizes to the $\mathrm{z}$-disc and interacts with desmin, vimentin, and actin $[59,60]$. Mutations in $\alpha B$ crystallin underlie myofibrillar myopathies and, crucially, the R120G mutation [61] has been shown to elevate autophagy in a transgenic mouse model [62].

As a tension bearer structure, misfolding of cytoskeletal crosslinkers and associated proteins might occur at the MTJ at higher rates than elsewhere in the fibre. Chaperon mediated degradation of damaged proteins has been linked to increased autophagy and protein turnover [43]. In addition to $\alpha \mathrm{B}$ crystallin, several chaperons were identified in the MTJ sample including Hspa8 (Hsc70). Given that Hspa8 has been shown to be involved in the chaperon mediated degradation of filamin $C$ [43], it is plausible that the presence of $\alpha \mathrm{B}$ crystallin and other chaperons in the MTJ sample relates to higher protein turnover in this localization. In conclusion, although purely descriptive, our results indicate that LCM and LC/MS-MS of myotendinous junction sections is a plausible experimental approach to identify novel factors involved in tension sensing and their regulation.

\section{Methods}

\section{Tissue processing}

Hind- and fore-limb muscles were dissected from mice and embedded into O.C.T. within small plastic biopsy molds (Sakura Finetek). The whole piece was immediately snap frozen in cold isopentane and stored at $-80^{\circ} \mathrm{C}$. Catapulting tests (see below) showed that sections of $20 \mu \mathrm{m}$ were optimal for LCM purification. Sections were placed on polyethylene naphthalate membrane slides (Leica Microsystems). The presence of myotendinous junction was checked every 3 slides, each slide containing 12 to $18 \mathrm{sec}-$ tions. A slight modification of the Hematoxylin and Gurr Eosin protocol (Santa Cruz) was used to stain the slides and facilitate the identification of the myotendionous junctions. Slides were incubated on hematoxylin for 1 minute and then washed under running water for 5 minutes. Eosin was then added just for 10 seconds, followed by a 5 minutes wash as before. Slides were then allowed to dry on an air cabinet overnight. Stained slides were used for LCM.

\section{LCM}

A laser pressure catapult protocol was applied using a PALM LCM inverted microscope (Zeiss). Membrane regions were marked and cut out by a focused laser beam by automated movement along a fixed laser focus. The dissected segments were then catapulted into an adhesive cap of a collection tube by a laser pulse. The volume of energy and focus was adjusted for the complete sectioning of myotendinous and extra junction membrane regions was: collagen rich myotendinous regions required $80 \%$ energy and $45 \%$ focus while extra junctional regions worked better with $75 \%$ energy and $42 \%$ focus. These values were also modified as required. Collected pieces were pelleted by brief centrifugation at $14000 \mathrm{~g}$ and kept at $-80^{\circ} \mathrm{C}$. All collected samples were pooled into single myotendinous junction and extrajunctional membrane pellets, resuspended into NuPAGE LDS sample buffer, heated at $70^{\circ} \mathrm{C}$ for 10 minutes and run on a Bis-Tris mini gel (Life technologies), before staining with Bio-Safe Coomassie stain (Bio-Rad).

\section{LC-MS/MS}

Gel sections were selected and excised. To minimise the potential for suppression from high abundance components; intensely stained bands were isolated from regions 
of low staining as shown in Figure 1B. In-gel tryptic digestion was performed after reduction with DTE and Scarbamidomethylation with iodoacetamide. Gel pieces were washed twice with $50 \%(\mathrm{v}: \mathrm{v})$ aqueous acetonitrile containing $25 \mathrm{mM}$ ammonium bicarbonate, then once with acetonitrile and dried in a vacuum concentrator for $20 \mathrm{~min}$. Sequencing-grade, modified porcine trypsin (Promega) was dissolved in the $50 \mathrm{mM}$ acetic acid supplied by the manufacturer, then diluted 5 -fold by adding $25 \mathrm{mM}$ ammonium bicarbonate to give a final trypsin concentration of $0.02 \mathrm{mg} / \mathrm{mL}$. Gel pieces were rehydrated by adding $10 \mu \mathrm{L}$ of trypsin solution, and after $30 \mathrm{~min}$ enough $25 \mathrm{mM}$ ammonium bicarbonate solution was added to cover the gel pieces. Digests were incubated overnight at $37^{\circ} \mathrm{C}$. Peptides were extracted from the gel by washing three times with $50 \%(\mathrm{v}: \mathrm{v})$ aqueous acetonitrile containing $0.1 \%$ trifluoroacetic acid (v:v), before being dried down in a vacuum concentrator and reconstituting in aqueous $0.1 \%$ (v:v) trifluoroacetic acid. Each section was digested and analysed by LC-MS/MS independently.

Samples were loaded onto a nanoAcquity UPLC system (Waters) equipped with a nanoAcquity Symmetry $\mathrm{C}_{18}$, $5 \mu \mathrm{m}$ trap $(180 \mu \mathrm{m} \times 20 \mathrm{~mm}$ Waters $)$ and a nanoAcquity BEH130 $1.7 \mu \mathrm{m} \mathrm{C}_{18}$ capillary column $(75 \mu \mathrm{m} \times 250 \mathrm{~mm}$, Waters). The trap wash solvent was $0.1 \%(\mathrm{v} / \mathrm{v})$ aqueous formic acid and the trapping flow rate was $10 \mu \mathrm{L} / \mathrm{min}$. The trap was washed for 5 min before switching flow to the capillary column. The separation used a gradient elution of two solvents (solvent A: $0.1 \%(\mathrm{v} / \mathrm{v})$ formic acid; solvent B: acetonitrile containing $0.1 \%(\mathrm{v} / \mathrm{v})$ formic acid). The flow rate for the capillary column was $300 \mathrm{~nL} / \mathrm{min}$ Column temperature was $60^{\circ} \mathrm{C}$ and the gradient profile was as follows: initial conditions $5 \%$ solvent $\mathrm{B}$, followed by a linear gradient to $30 \%$ solvent B over $125 \mathrm{~min}$, then a linear gradient to $50 \%$ solvent B over $5 \mathrm{~min}$, followed by a wash with $95 \%$ solvent B for $10 \mathrm{~min}$. The column was returned to initial conditions and re-equilibrated for $30 \mathrm{~min}$ before subsequent injections.

The nanoLC system was interfaced with a maXis LCMS/MS System (Bruker Daltonics) with a Bruker nanoelectrospray source fitted with a steel emitter needle $(180 \mu \mathrm{m}$ O.D. $\times 30 \mu \mathrm{m}$ I.D., Thermo (Proxeon)). Positive ESI- MS \& MS/MS spectra were acquired using AutoMSMS mode. Instrument control, data acquisition and processing were performed using Compass 1.3 SR3 software (microTOF control, Hystar and DataAnalysis, Bruker Daltonics). Instrument settings were: ion spray voltage: $1,400 \mathrm{~V}$, dry gas: $4 \mathrm{~L} / \mathrm{min}$, dry gas temperature $160^{\circ} \mathrm{C}$, ion acquisition range: $m / z 50-2,200$. AutoMSMS settings were: MS: $0.5 \mathrm{~s}$ (acquisition of survey spectrum), MS/MS (CID with $\mathrm{N}_{2}$ as collision gas): ion acquisition range: $\mathrm{m} / z$ $300-1,500,0.1 \mathrm{~s}$ acquisition for precursor intensities above 100,000 counts, for signals of lower intensities down to 1,000 counts acquisition time increased linear to $1 \mathrm{~s}$, the collision energy and isolation width settings were automatically calculated using the AutoMSMS fragmentation table: 8 precursor ions, absolute threshold 1,000 counts, preferred charge states: $2-4$, singly charged ions excluded. $1 \mathrm{MS} / \mathrm{MS}$ spectrum was acquired for each precursor and former target ions were excluded for $30 \mathrm{~s}$.

\section{Database search}

Spectra were calibrated using a lock mass signal $(\mathrm{m} / \mathrm{z}$ 1221.99064) prior to compound detection and peak list creation. The peak list files obtained from individual gel sections were combined and then submitted for database searching to a locally-running copy of the Mascot program (Matrix Science Ltd., version 2.3.02), through the ProteinScape interface (Bruker Daltonics., version 2.1). The database searched was IPI.mouse (v3.87 27/11/2011). Search criteria included: enzyme, trypsin; missed cleavages, 1; fixed modifications, carbamidomethyl (C); variable modifications, acetyl (N-terminal) and oxidation (M); peptide tolerance, $10 \mathrm{ppm}$; MS/MS tolerance, $0.1 \mathrm{Da}$. The search included an automatic decoy database search and the false discovery rate for identity was $<2 \%$. The significance threshold was $\mathrm{p}<0.05$ and the peptide ion score cut-off was 20 .

\section{Immunofluorescence}

Tissues were obtained from 30 to 45 days old C57BL/6 male mice killed by cervical dislocation. Tissues were frozen in isopentane cooled in liquid nitrogen prior to cryosectioning and then stored at $-80^{\circ} \mathrm{C}$. After thawing and drying, sections were fixed with either cold acetone (pure or as a 1:1 mix with cold methanol) or $4 \%$ paraformaldehyde for $30 \mathrm{~min}$ and rinsed with PBS three times or used unfixed. Standard indirect immunohistochemistry was then employed using primary antibodies followed by FITC conjugated polyclonal anti-mouse, -rabbit or -goat secondary antibodies using dilutions as recommended by the manufacturer (Sigma-Aldrich). The primary antibodies used in this work were: goat anti human FHL1 (cat. AHP2070, AbD Serotec), mouse monoclonal anti-syntrophin (cat. SAB4200213, Sigma-Aldrich), mouse monoclonal antimyomesin (cat. mMaC myomesin B4, DSHB), mouse monoclonal anti-titin (cat. 9 D10, DSHB), mouse monoclonal anti s-laminin (cat. C4, DSHB), mouse monoclonal anti filaminC RR90 (an IgA sub-type [31]), mouse monoclonal anti-Annexin I (cat. EH17a, DSHB), mouse monoclonal anti-myotilin (RSO34, Novocastra), mouse monoclonal anti-desmin (cat. D76, DSHB), rabbit polyclonal anti-TACP (cat. QC18385, Sigma-Aldrich), mouse monoclonal anti- $\alpha \mathrm{B}$ crystallin (cat. CPTC-CRYAB-3, DSHB), mouse monoclonal anti-tubulin (cat. E7, DSHB), mouse monoclonal anti- $\alpha$ actinin (cat EA53, Sigma-Aldrich), mouse monoclonal anti- $\alpha$-sarcoglycan (cat. IVD3(1)A9, DSHB). Initial dilutions were at 1:200 in all cases and adjusted accordingly 
depending on the signal to noise. At the dilutions used, none of the secondary antibodies used on their own gave a detectable signal using identical incubation conditions and confocal settings (data not shown). All images were taken with a Zeiss 510 upright confocal microscope using a plan-Apochromat $63 \times / 1.4$ Oil DIC lense at $1024 \times 1024$ resolution. Detailed views of regions of interest were obtained using various zoom factors as required, using the same resolution. ImageJ (http://imagej.nih.gov/ij/index. $\mathrm{html}$ ) was used to merge channels and produce the figures.

\section{Additional files}

Additional file 1: Table S1. Mouse proteins identified in myotendinous junction (MTJ) and peripheral membrane (M) LCM samples. Individual lists are ranked by Mascot score. The 'Rank' entry refers to protein's ranking in the original Mascot results for each sample.

Additional file 2: Figure S1. Blind analysis of the most likely cellular components associated with the proteins identified by LC-MS/MS in the myotendinous junction. A diagram generated by the GOrilla software (see text for details) using all proteins identified in the myotendinous junction sample. The color of the box indicates $P$ value interval of the enclosed term: white, yellow, orange, brown and red denotes $P$ values $>10^{-3}, 10^{-3}$ to $10^{-5}$, $10^{-5}$ to $10^{-7}, 10^{-7}$ to $10^{-9}$ and $<10^{-9}$ respectively. The $P$ value is the enrichment $\mathrm{p}$-value computed according to a minimum hypergeometric model and is not corrected for multiple testing (see Additional file 3: Table S2 for corrected $P$ values).

Additional file 3: Table S2. Full lists of genes per enriched $G O$ term with the corrected $p$ values* for multiple testing [30].

\section{Abreviations}

MTJ: (myotendinous junction); M: (extra junctional membrane); LCM: (laser capture microscopy); LC/MS-MS: (liquid chromatography tandem mass spectrometry); GO: (gene ontology); DAPC: (dystrophin-associated protein complex).

\section{Competing interests}

The authors declare that they have no competing interests.

\section{Authors' contributions}

TC carried our laser dissections from sections; LF carried out histological preparations and immunostanings; DA carried out LCM/MS/MS; AD carried out SDS gel processing and trypsin digestions; JT provided advice on methodology and literature review; PO provided confocal support for revised manuscript; GB design the experimental plan and wrote the article. All authors read and approved the final manuscript.

\section{Acknowledgements}

This work was part-funded by the Wellcome Trust [ref: 097829] through the Centre for Chronic Diseases and Disorders (C2D2) at the University of York.

\section{Author details}

'Department of Biology, University of York, Wentworth Way, York YO10 5DD, UK. ${ }^{2}$ Bioscience Technology Facility, Department of Biology, University of York, Wentworth Way, York YO10 5DD, UK.

\section{Received: 20 September 2013 Accepted: 25 April 2014}

Published: 7 May 2014

\section{References}

1. Micke P, Ostman A, Lundeberg J, Ponten F: Laser-assisted cell microdissection using the PALM system. Methods Mol Biol 2005, 293:151-166.

2. Schermelleh L, Thalhammer S, Heckl W, Posl H, Cremer T, Schutze K, Cremer M: Laser microdissection and laser pressure catapulting for the generation of chromosome-specific paint probes. Biotechniques 1999, 27(2):362-367.

3. Kolble K: The LEICA microdissection system: design and applications. J Mol Med (Berl) 2000, 78(7):B24-25.
4. Buckanovich RJ, Sasaroli D, O'Brien-Jenkins A, Botbyl J, Conejo-Garcia JR, Benencia F, Liotta LA, Gimotty PA, Coukos G: Use of immuno-LCM to identify the in situ expression profile of cellular constituents of the tumor microenvironment. Cancer Biol Ther 2006, 5(6):635-642.

5. Nazarian J, Bouri K, Hoffman EP: Intracellular expression profiling by laser capture microdissection: three novel components of the neuromuscular junction. Physiol Genomics 2005, 21(1):70-80.

6. Ketterer C, Zeiger U, Budak MT, Rubinstein NA, Khurana TS: Identification of the neuromuscular junction transcriptome of extraocular muscle by laser capture microdissection. Invest Ophthalmol Vis Sci 2010, 51(9):4589-4599.

7. Xu BJ: Combining laser capture microdissection and proteomics: methodologies and clinical applications. Proteomics Clin Appl 2010 4(2):116-123.

8. Liao L, Cheng D, Wang J, Duong DM, Losik TG, Gearing M, Rees HD, Lah JJ, Levey Al, Peng J: Proteomic characterization of postmortem amyloid plaques isolated by laser capture microdissection. J Biol Chem 2004, 279(35):37061-37068.

9. Leverenz JB, Umar I, Wang Q, Montine TJ, McMillan PJ, Tsuang DW, Jin J, Pan C, Shin J, Zhu D, Zhang J: Proteomic identification of novel proteins in cortical lewy bodies. Brain Pathol 2007, 17(2):139-145.

10. Wang Q, Woltjer RL, Cimino PJ, Pan C, Montine KS, Zhang J, Montine TJ: Proteomic analysis of neurofibrillary tangles in Alzheimer disease identifies GAPDH as a detergent-insoluble paired helical filament tau binding protein. FASEB J 2005, 19(7):869-871.

11. Zellner $M$, Veitinger $M$, Umlauf $E$ : The role of proteomics in dementia and Alzheimer's disease. Acta Neuropathol 2009, 118(1):181-195.

12. Kurapati R, McKenna C, Lindqvist J, Williams D, Simon M, LeProust E, Baker J, Cheeseman M, Carroll N, Denny P, Laval S, Lochmüller H, Ochala J, Blanco G: Myofibrillar myopathy caused by a mutation in the motor domain of mouse MyHC Ilb. Hum Mol Genet 2012, 21(8):1706-1724.

13. Roulhac PL, Ward JM, Thompson JW, Soderblom EJ, Silva M, Moseley MA III, Jarvis ED: Microproteomics: quantitative proteomic profiling of small numbers of laser-captured cells. Cold Spring Harb Protoc 2011, (2): doi:10.1101/pdb.prot5573.

14. Charvet B, Ruggiero F, Le Guellec D: The development of the myotendinous junction. A review. Muscl Ligaments Tendons J 2012, 2(2):53-63.

15. Tidball JG: Myotendinous junction: morphological changes and mechanical failure associated with muscle cell atrophy. Exp Mol Pathol 1984, 40(1):1-12.

16. Pardo JV, Siliciano JD, Craig SW: A vinculin-containing cortical lattice in skeletal muscle: transverse lattice elements ("costameres") mark sites of attachment between myofibrils and sarcolemma. Proc Natl Acad Sci U S A 1983, 80(4):1008-1012.

17. Volk T, Fessler $\mathrm{LI}$, Fessler $\mathrm{JH}$ : A role for integrin in the formation of sarcomeric cytoarchitecture. Cell 1990, 63(3):525-536.

18. Kadrmas JL, Beckerle MC: The LIM domain: from the cytoskeleton to the nucleus. Nat Rev Mol Cell Biol 2004, 5(11):920-931.

19. Reedy MC, Beall C: Ultrastructure of developing flight muscle in Drosophila. II Formation of the myotendon junction. Dev Biol 1993, 160(2):466-479.

20. Ervasti JM: Costameres: the Achilles' heel of Herculean muscle. J Biol Chem 2003, 278(16):13591-13594.

21. Kojima H, Sakuma E, Mabuchi Y, Mizutani J, Horiuchi O, Wada I, Horiba M, Yamashita Y, Herbert DC, Soji T, Otsuka T: Ultrastructural changes at the myotendinous junction induced by exercise. J Orthop Sci 2008, 13(3):233-239.

22. Lek M, North KN: Are biological sensors modulated by their structural scaffolds? The role of the structural muscle proteins alpha-actinin-2 and alpha-actinin-3 as modulators of biological sensors. FEBS Lett 2010, 584(14):2974-2980

23. Knoll R, Buyandelger B, Lab M: The sarcomeric Z-disc and Z-discopathies. J Biomed Biotechnol 2011, 2011:569628.

24. Frank D, Frey N: Cardiac Z-disc signaling network. J Biol Chem 2011, 286(12):9897-9904.

25. Baker J, Riley G, Romero MR, Haynes AR, Hilton H, Simon M, Hancock J, Tateossian H, Ripoll VM, Blanco G: Identification of a Z-band associated protein complex involving KY, FLNC and IGFN1. Exp Cell Res 2010, 316(11):1856-1870.

26. Blanco G, Coulton GR, Biggin A, Grainge C, Moss J, Barrett M, Berquin A, Marechal G, Skynner M, van Mier P, Nikitopoulou A, Kraus M, Ponting CP, Mason RM, Brown SD: The kyphoscoliosis (ky) mouse is deficient in 
hypertrophic responses and is caused by a mutation in a novel muscle-specific protein. Hum Mol Genet 2001, 10(1):9-16.

27. Granger BL, Lazarides E: The existence of an insoluble $Z$ disc scaffold in chicken skeletal muscle. Cell 1978, 15(4):1253-1268.

28. Luther PK: The vertebrate muscle Z-disc: sarcomere anchor for structure and signalling. J Muscle Res Cell Motil 2009, 30(5-6):171-185.

29. Eden E, Navon R, Steinfeld I, Lipson D, Yakhini Z: GOrilla: a tool for discovery and visualization of enriched $\mathrm{GO}$ terms in ranked gene lists. BMC Bioinformatics 2009, 10:48.

30. Benjamini $Y$, Hochberg $Y$ : Controlling the False Discovery Rate: A Practical and Powerful Approach to Multiple Testing. J Royal Stat Soc Series B (Methodological) 1995, 57(1):289-300.

31. van der Ven PF, Obermann WM, Lemke B, Gautel M, Weber K, Furst DO: Characterization of muscle filamin isoforms suggests a possible role of gamma-filamin/ABP-L in sarcomeric Z-disc formation. Cell Motil Cytoskeleton 2000, 45(2):149-162.

32. Tidball JG: Desmin at myotendinous junctions. Exp Cell Res 1992 199(2):206-212.

33. Atsuta F, Sato K, Maruyama K, Shimada Y: Distribution of connectin (titin) nebulin and alpha-actinin at myotendinous junctions of chicken pectoralis muscles: an immunofluorescence and immunoelectron microscopic study. J Muscle Res Cell Motil 1993, 14(5):511-517.

34. Masuda T, Fujimaki N, Ozawa E, Ishikawa H: Confocal laser microscopy of dystrophin localization in guinea pig skeletal muscle fibers. J Cell Biol 1992, 119(3):543-548.

35. Samitt CE, Bonilla E: Immunocytochemical study of dystrophin at the myotendinous junction. Muscle Nerve 1990, 13(6):493-500,

36. Agarkova I, Ehler E, Lange S, Schoenauer R, Perriard JC: M-band: a safeguard for sarcomere stability? J Muscle Res Cell Motil 2003, 24(2-3):191-203.

37. Schoenauer R, Lange S, Hirschy A, Ehler E, Perriard JC, Agarkova I: Myomesin 3, a novel structural component of the M-band in striated muscle. J Mol Biol 2008, 376(2):338-351.

38. Knoll R, Hoshijima M, Hoffman HM, Person V, Lorenzen-Schmidt I, Bang ML, Hayashi T, Shiga N, Yasukawa H, Schaper W, McKenna W, Yokoyama M, Schork NJ, Omens JH, McCulloch AD, Kimura A, Gregorio CC, Poller W, Schaper J, Schultheiss HP, Chien KR: The cardiac mechanical stretch sensor machinery involves a $\mathbf{Z}$ disc complex that is defective in a subset of human dilated cardiomyopathy. Cell 2002, 111(7):943-955.

39. Arimura T, Ishikawa T, Nunoda S, Kawai S, Kimura A: Dilated cardiomyopathy-associated BAG3 mutations impair Z-disc assembly and enhance sensitivity to apoptosis in cardiomyocytes. Hum Mutat 2011, 32(12):1481-1491.

40. Xu BJ, Caprioli RM, Sanders ME, Jensen RA: Direct analysis of laser capture microdissected cells by MALDI mass spectrometry. J Am Soc Mass Spectrom 2002, 13(11):1292-1297.

41. Senga K, Kobayashi M, Hattori H, Yasue K, Mizutani H, Ueda M, Hoshino T: Type VI collagen in mouse masseter tendon, from osseous attachment to myotendinous junction. Anat Rec 1995, 243(3):294-302.

42. Fichard A, Kleman JP, Ruggiero F: Another look at collagen V and XI molecules. Matrix Biol 1995, 14(7):515-531.

43. Ulbricht A, Eppler FJ, Tapia VE, van der Ven PF, Hampe N, Hersch N, Vakeel P, Stadel D, Haas A, Saftig P, Behrends C, Fürst DO, Volkmer R, Hoffmann B, Kolanus W, Höhfeld J: Cellular mechanotransduction relies on tensioninduced and chaperone-assisted autophagy. Curr Biol 2013, 23(5):430-435.

44. Arndt V, Dick N, Tawo R, Dreiseidler M, Wenzel D, Hesse M, Furst DO, Saftig P, Saint R, Fleischmann BK, Hoch M, Höhfeld J: Chaperone-assisted selective autophagy is essential for muscle maintenance. Curr Biol 2010, 20(2):143-148.

45. Ehrlicher AJ, Nakamura F, Hartwig JH, Weitz DA, Stossel TP: Mechanical strain in actin networks regulates FilGAP and integrin binding to filamin A. Nature 2011, 478(7368):260-263.

46. Tskhovrebova L, Trinick J: Titin: properties and family relationships. Nat Rev Mol Cell Biol 2003, 4(9):679-689.

47. Kenny PA, Liston EM, Higgins DG: Molecular evolution of immunoglobulin and fibronectin domains in titin and related muscle proteins. Gene 1999, 232(1):11-23.

48. Agarkova I, Perriard JC: The M-band: an elastic web that crosslinks thick filaments in the center of the sarcomere. Trends Cell Biol 2005, 15(9):477-485.

49. Schoenauer R, Bertoncini P, Machaidze G, Aebi U, Perriard JC, Hegner M, Agarkova I: Myomesin is a molecular spring with adaptable elasticity. J Mol Biol 2005, 349(2):367-379
50. von Nandelstadh P, Gronholm M, Moza M, Lamberg A, Savilahti H, Carpen O: Actin-organising properties of the muscular dystrophy protein myotilin. Exp Cell Res 2005, 310(1):131-139.

51. Hauser MA, Horrigan SK, Salmikangas P, Torian UM, Viles KD, Dancel R, Tim RW, Taivainen A, Bartoloni L, Gilchrist JM, Stajich JM, Gaskell PC, Gilbert JR, Vance JM, Pericak-Vance MA, Carpen O, Westbrook CA, Speer MC: Myotilin is mutated in limb girdle muscular dystrophy 1A. Hum Mol Genet 2000, 9(14):2141-2147.

52. Selcen D, Engel AG: Mutations in myotilin cause myofibrillar myopathy. Neurology 2004, 62(8):1363-1371.

53. Foroud T, Pankratz N, Batchman AP, Pauciulo MW, Vidal R, Miravalle L, Goebel HH, Cushman L, Azzarelli B, Horak H, Farlow M, Nichols WC: A mutation in myotilin causes spheroid body myopathy. Neurology 2005, 65(12):1936-1940.

54. Ernst JD, Hoye E, Blackwood RA, Mok TL: Identification of a domain that mediates vesicle aggregation reveals functional diversity of annexin repeats. J Biol Chem 1991, 266(11):6670-6673.

55. Monastyrskaya K, Babiychuk EB, Hostettler A, Wood P, Grewal T, Draeger A: Plasma membrane-associated annexin $\mathrm{A} 6$ reduces $\mathrm{Ca} 2+$ entry by stabilizing the cortical actin cytoskeleton. J Biol Chem 2009, 284(25):17227-17242.

56. Mishra S, Chander V, Banerjee P, Oh JG, Lifirsu E, Park WJ, Kim do H, Bandyopadhyay A: Interaction of annexin A6 with alpha actinin in cardiomyocytes. BMC Cell Biol 2011, 12:7.

57. Bizzarro V, Fontanella B, Franceschelli S, Pirozzi M, Christian H, Parente L, Petrella A: Role of Annexin A1 in mouse myoblast cell differentiation. J Cell Physiol 2010, 224(3):757-765.

58. Dubin RA, Ally AH, Chung S, Piatigorsky J: Human alpha B-crystallin gene and preferential promoter function in lens. Genomics 1990, 7(4):594-601.

59. Bennardini F, Wrzosek A, Chiesi M: Alpha B-crystallin in cardiac tissue. Association with actin and desmin filaments. Circul Res 1992. 71(2):288-294.

60. Nicholl ID, Quinlan RA: Chaperone activity of alpha-crystallins modulates intermediate filament assembly. EMBO f 1994, 13(4):945-953.

61. Vicart P, Caron A, Guicheney P, Li Z, Prevost MC, Faure A, Chateau D, Chapon F, Tome F, Dupret JM, Paulin D, Fardeau M: A missense mutation in the alphaB-crystallin chaperone gene causes a desmin-related myopathy. Nat Genet 1998, 20(1):92-95.

62. Zheng Q, Su H, Ranek MJ, Wang X: Autophagy and p62 in cardiac proteinopathy. Circ Res 2011, 109(3):296-308.

doi:10.1186/1477-5956-12-25

Cite this article as: Can et al:: Proteomic analysis of laser capture microscopy purified myotendinous junction regions from muscle sections. Proteome Science 2014 12:25.

\section{Submit your next manuscript to BioMed Central and take full advantage of:}

- Convenient online submission

- Thorough peer review

- No space constraints or color figure charges

- Immediate publication on acceptance

- Inclusion in PubMed, CAS, Scopus and Google Scholar

- Research which is freely available for redistribution 